\title{
REVIEW \\ Placental growth factor and pre-eclampsia
}

\author{
$\mathrm{KChau}^{1,2,3}$, A Hennessy ${ }^{1,3,4}$ and A Makris ${ }^{1,4,5,6}$
}

Placental growth factor (PIGF) is an increasingly important molecule in the prediction, diagnosis and treatment of pre-eclampsia. It has pro-angiogenic effects on the feto-placental circulation and supports trophoblast growth. Mechanisms by which PIGF expression is regulated continue to be investigated. Low circulating PIGF precedes the manifestation of clinical disease in preeclamptic pregnancies and intrauterine growth restriction. This suggests that low PIGF is a marker of abnormal placentation, but it remains uncertain whether this is a cause or consequence. Prediction of pre-eclampsia using PIGF is promising and may assist in the targeting of resources to women at highest risk of adverse pregnancy outcomes. Promisingly, experimental animal models of preeclampsia have been successfully treated with supplemental PIGF. Treatment of pre-eclampsia with PIGF is a potential therapeutic option requiring further exploration. This review focuses specifically on the role of PIGF in normal and pathological placental development and in the clinical management of pre-eclampsia.

Journal of Human Hypertension (2017) 31, 782-786; doi:10.1038/jhh.2017.61; published online 24 August 2017

\section{INTRODUCTION}

Placental growth factor (PIGF) is an increasingly important player in the clinical management of pre-eclampsia. This review outlines the role of PIGF in human physiology but focuses specifically on the current understanding of PIGF's function in normal and pathological placental development. The differences in peripheral concentrations of PIGF between normal and pre-eclamptic pregnancy are highlighted and utility of PIGF as a predictive or diagnostic test for pre-eclampsia is discussed. Finally, the possibility of PIGF as treatment for pre-eclampsia is considered.

\section{PLGF}

Placental growth factor is a member of the vascular endothelial growth factor (VEGF) family and is predominantly expressed in the placenta, although it is also expressed at low levels in many other tissues, including the heart, lung, thyroid, liver, skeletal muscle and bone. The human PIGF gene is located on chromosome $14 q 14$ and encodes 4 isoforms of PIGF. The protein is secreted as a glycosylated homodimer and PIGF-1 and -3 are diffusible isoforms whereas PIGF-2 and PIGF-4 have heparin binding domains. Of these, PIGF-1 and -2 are the most abundant forms, and during pregnancy they are secreted in a strongly correlated manner, indicating a common regulation mechanism. The presence of a heparin binding domain suggests that PIGF-2 and -4 remain cell membrane-associated and act in an autocrine fashion, while the diffusible forms of PIGF probably affect targets in a paracrine manner. Mice produce only the PIGF-2 variant. Placental growth factor binds to VEGFR-1 (vascular endothelial growth factor-1 receptor-1) or FLT-1 (fms-related tyrosine kinase-1) and its soluble variant SFLT-1 (soluble fms-like tyrosine kinase-1), but not VEGFR-2 (vascular endothelial growth factor receptor-2), also known as KDR (kinase insert domain receptor) or FLK-1 (foetal liver kinase-1) (Figure 1). It also binds to neuropilin receptor-1 (NP-1) and -2 present in neurons. NP-1 has also recently been identified in placenta but its role is yet to be elucidated. ${ }^{1}$

\section{PLGF AND ANGIOGENESIS}

Angiogenesis is a vital process for embryonic development and growth is regulated by a complex interplay of a multitude of factors, including the VEGF family. New blood vessels form by remodelling of existing vasculature with sprouting of new branches followed by non-branching angiogenesis-elongation and enlargement. In human adult life, angiogenesis occurs predominantly in the endometrium during the menstrual cycle, in wound healing and as an adaptive process in the myocardium and skeletal muscle. Placental growth factor is pro-angiogenic as it enhances the activity of VEGF by competitively binding to the VEGFR-1 receptor, allowing VEGF to bind then to VEGFR-2 which has stronger tyrosine kinase activity. However, PIGF also exerts it's affect through other mechanisms such as intermolecular transphosphorylation of VEGFR-2 following activation of VEGFR-1, which amplifies VEGFR-2 response to VEGF binding. Additionally, PIGF forms a heterodimer with VEGF, which may have either proor anti-angiogenic effects.

The main role of PIGF in tissues other than the placenta is angiogenesis in response to pathological ischaemia or injury. Knockout mice $\left(\mathrm{PIGF}^{-/-}\right)$have impaired angiogenesis and arteriogenesis during pathological conditions such as heart, limb and ocular ischaemia. Physiological exercise induced ischaemia in skeletal and cardiac muscle does not stimulate PIGF production but PIGF is upregulated in pathological conditions such as coronary artery disease. Accordingly, PIGF expression is upregulated by hypoxia in non-trophoblast cells. ${ }^{2}$ In contrast, transcriptional activity of PIGF in trophoblast is suppressed by hypoxia ${ }^{3}$ and increased by a normoxic environment pointing to a specific regulatory mechanism and function in these cells. ${ }^{2}$ 


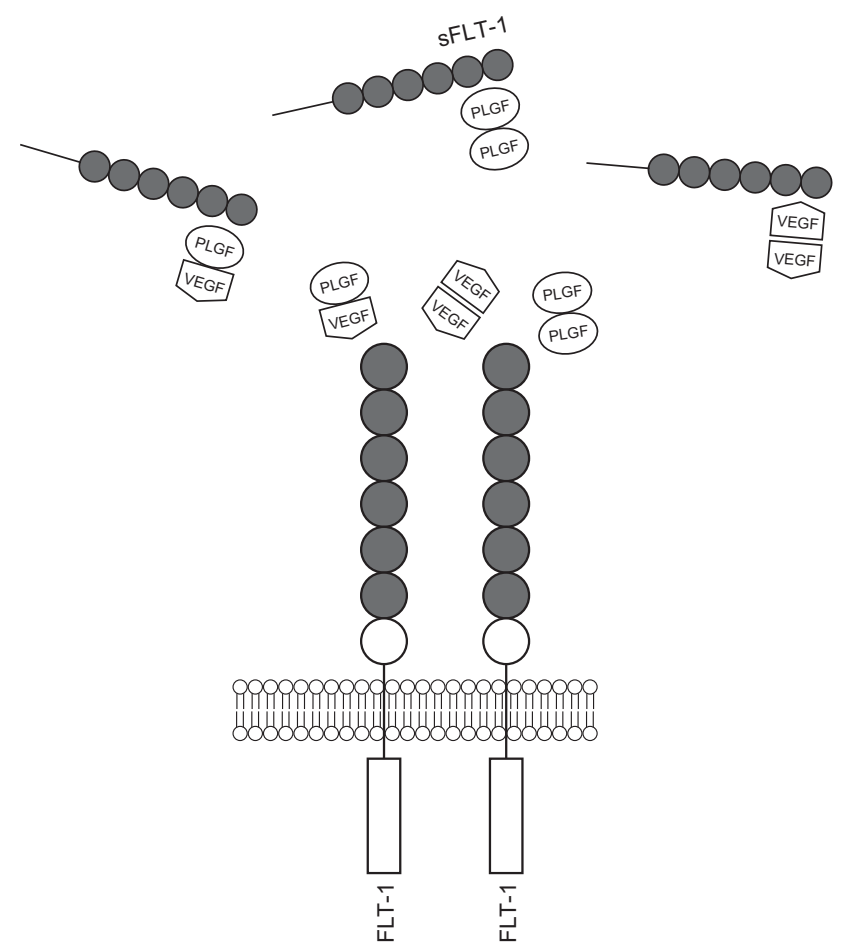

Figure 1. Interaction between VEGF, PIGF, FLT-1 and SFLT-1. VEGF and PIGF homodimers and heterodimers bind to FLT-1 and SFLT-1.

In tumour cells, PIGF expression is part of the angiogenic switch that supports tumour vascularisation. In most cancers (solid and haematological), there is a positive correlation between cancer severity and PIGF expression and protein levels, with an inverse relationship between PIGF and survival. The role of PIGF is context dependent, varying according to tumour type and stage and consequently tumour reduction with PIGF inhibition has not been demonstrated consistently. For example, overexpression of PIGF can decrease rather than increase angiogenesis as formation of VEGF/PLGF heterodimers are less pro-angiogenic than VEGF homodimers ${ }^{4}$ and, in another study, blockade of PIGF seemed to improve the tumour vasculature quality. ${ }^{5}$ The specific expression of PIGF in diseased tissues presents itself as a good opportunity for targeted therapy. A phase 1 study of an antiPIGF monoclonal antibody in combination with anti-VEGF antibody bevacizumab in patients with recurrent glioblastoma multiforme did not show improved survival. ${ }^{6}$ Two further studies have been conducted in patients with hepatocellular carcinoma and metastatic colorectal or ovarian cancer but results have not been reported.

\section{PLGF IN REPRODUCTION}

The role of PIGF in reproduction is still being understood. PIGF is thought to be redundant in reproduction as PIGF knockout mice are fertile and pups have similar growth potential compared to wild type mice. However, endometrial tissue during the secretory phase of the human menstrual cycle has been shown to secrete PIGF.7 The presence of PIGF during this window supports a role of PIGF in influencing embryo implantation, but this has yet to be further characterised. Although PLGF knockout mice appear normal, differences in foetal and adult brain development ${ }^{8}$ have recently been demonstrated. The preliminary data in the children of pre-eclamptic women suggest subtle differences in brain vascular development, which is thought to be related to intrauterine events. ${ }^{9}$ Therefore, while PIGF may not be essential to reproduction it is still likely to be an important influence on pregnancy and vascular development.

\section{THE ROLE OF PLGF IN PLACENTAL DEVELOPMENT}

Circulating PIGF is prominently elevated in pregnancy with the source being the placenta. The function of PIGF in the placenta is likely to be in the promotion of development and maturation of the placental vascular system.

Implantation sites of PIGF knockout mice show abnormal placental vasculature. ${ }^{10}$ There is decreased branching in the antimesometrial (feto-placental) vessels and increased lacunarity, indicating a lack of uniformity of vessel formation. Uteroplacental vessels also display decreased branching, but decidual invasion is not influenced. Mouse uterine lymphatic vessels are also abnormally developed. In human placenta, expression of PIGF corresponds with different stages in placental development with non-branching angiogenesis of the feto-placental circulation and maturation of the utero-placental circulation coinciding with increased expression of PIGF in later gestation. Evidently, development of the placental circulation is influenced by PIGF even though the absence of this angiogenic factor does not result in death in offspring of knockout mice. In contrast, a mouse knockin with PIGF expressed in T cells showed decreased angiogenesis in offspring, ${ }^{11}$ suggesting that, analogous to the variable role of PIGF in a tumour life cycle, the effect of PIGF in pregnancy is situation specific.

When postulating possible effects in human pregnancy from the findings in rodent studies, differences in placental physiology between species must be noted. Although abnormal implantation evident in PIGF knockout mice may not lead to embryonic or foetal death, the consequences of aberrant placental development may not be readily apparent in mice. Unlike human pregnancy, mouse placenta does not invade into the myometrium and endovascular invasion is limited. Consequently, abnormal spiral artery remodelling in mice does not lead to placental insufficiency or abnormal blood pressure regulation. ${ }^{12}$ Processes in human placenta development may be more extensively mediated by PIGF.

Placental expression of PIGF dominates from the second trimester when the utero-placental circulation is advancing, with myometrial spiral arteries remodelling in a 'second wave' of invasion beginning at 16-18 weeks' gestation. However, there have been conflicting reports as to whether PIGF contributes to trophoblast invasion. ${ }^{13,14}$ Trophoblast develop invasive characteristics in response to increased oxygen tension and PIGF expression also increases with improved placental oxygenation, but it is uncertain whether these two events have a directly linked regulatory mechanism. Differentiation of uterine natural killer cells is influenced by PIGF ${ }^{15}$ and these cells may in turn mediate trophoblast invasion into the decidua.

PIGF increases proliferation of trophoblast cells. It also reduces apoptosis of trophoblast cells ${ }^{16}$ when these cells are starved, but not when they are exposed to inflammatory cytokines. ${ }^{17}$ This may manifest as increased circulating trophoblast debris found in cases of pre-eclampsia (where there is often PIGF deficiency), but the exact role of PIGF mediated reduction of apoptosis in placental development is not clear.

\section{PLGF LEVELS IN NORMAL PREGNANCY}

Concentrations of PIGF are low in the first trimester of an uncomplicated pregnancy and increases from week 11 to 12 onwards to a peak at week 30, after which it decreases (Figure 2). This is in contrast with sFLT-1, which increases towards the completion of pregnancy. This normal divergence of angiogenic factors levels occurs as the bioavailability of PIGF is reduced by binding to sFLT-1. Normal PIGF concentrations are dependent on 


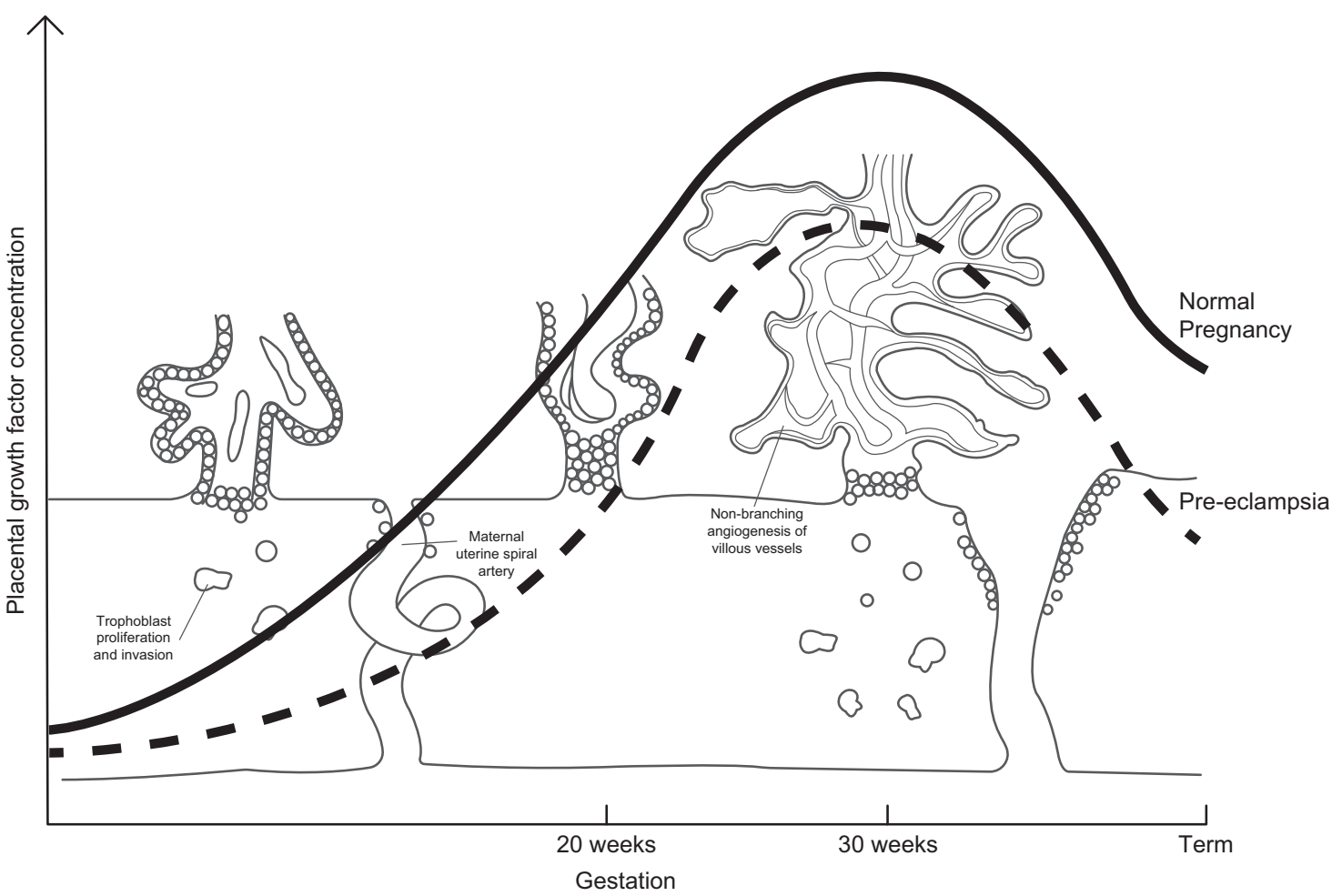

Figure 2. Circulating PIGF concentrations gradually increase during pregnancy to reach a peak at $\sim 30$ weeks gestation. In pre-eclampsia PIGF concentrations are comparatively lower throughout pregnancy. Placental expression of PIGF dominates from the second trimester of pregnancy, coinciding with non-branching angiogenesis of feto-placental vessels and maturation of the utero-placental circulation. Placental growth factor may contribute to trophoblast invasion, increase trophoblast proliferation and reduce apoptosis.

gestational age, with the lower limit of normal (defined as the 5 th centile) ranging from a peak of approximately $141 \mathrm{pg} \mathrm{m}^{-1}$ at around 30 weeks gestation to $23 \mathrm{pg} \mathrm{ml}^{-1}$ at term. ${ }^{18}$

\section{PLGF IN PRE-ECLAMPSIA}

Serum and urinary PIGF is found to be decreased in women both at the time of diagnosis with pre-eclampsia and well in advance of syndrome onset. The deficiency in PIGF is likely due to a combination of decreased expression of PIGF and reduced free PIGF due to binding with sFLT-1, which is elevated in affected women. ${ }^{19}$ In early pregnancy, PIGF concentrations are lower in women who subsequently develop preclampsia than in normal pregnant women, but SFLT-1 levels are no different, suggesting that PIGF expression in the placenta is decreased. However, towards completion of pregnancy, there is a reciprocal relationship between SFLT-1 and PIGF with rising levels of total (free and bound to VEGF or PIGF) sFLT-1 and lower free PIGF levels. ${ }^{20}$ This suggests that in the latter half of pregnancy, low PIGF concentrations occur predominantly due to sequestering of PIGF by SFLT-1.

Low circulating PIGF is probably both a consequence of abnormal early events in placentation and a contributing factor to continued abnormal growth during the latter half of pregnancy. The hypothesis that PIGF is an indicator of abnormal placentation is supported by the observation that women without preeclampsia who give birth to small for gestational age babies also have low PIGF early in pregnancy. ${ }^{21}$ The data regarding the expression of PIGF in placental tissue is conflicting. Expression of PIGF is postulated to be lowered due to suppression by persistent placental hypoxia resulting from an underdeveloped uteroplacental circulation. However, studies have also shown increased $^{3}$ or no change in PIGF expression ${ }^{22}$ in pre-eclamptic placental tissue. Regulation of PIGF expression is unclear, but several mechanisms have been explored such as endoplasmic reticulum stress and epigenetic changes altering the effect of the transcription factor hypoxia-inducible factor-1a (HIF1-a), although the role of HIF1-a in trophoblast growth is debated. ${ }^{2,23,24}$ Inflammation may also influence PIGF expression as PIGF concentrations are elevated in sepsis.

\section{PLGF FOR THE PREDICTION AND DIAGNOSIS OF PRE- ECLAMPSIA}

Despite intense research efforts, the diagnosis and management pre-eclampsia has remained unchanged for decades. Recognition of the differences in circulating angiogenic factor levels between pre-eclamptic and normal pregnancies has resulted in investigation into whether these factors can identify women who require close monitoring. Once the diagnosis of pre-eclampsia is made, only delivery of the placenta can alleviate the condition.

In women who will develop pre-eclampsia, PIGF is low in the first trimester, well before the disease clinically manifests. Despite the differences between groups, single angiogenic factors are not useful for prediction ${ }^{25}$ with PIGF having a sensitivity of $32 \%$ for a $5 \%$ false-positive rate. Combinations of angiogenic factors such as the sFLT-1:PIGF ratio with aspects of history or ultrasound findings to create multifactorial predictive tools are promising but not currently in wide usage. For example the Fetal Medicine Foundation predictive algorithm at $11-13$ weeks gestation, ${ }^{26}$ which uses a combination of maternal characteristics, mean arterial pressure, uterine artery pulsatility index, PAPP-A and PIGF detects 95 and $46 \%$ of women with early and late pre-eclampsia, respectively with a false-positive rate of $10 \% .{ }^{27}$ Application of prediction algorithms in specific subgroups such as women with antiphospholipid syndrome and systemic lupus erythematosus may be more successful due to the increased baseline risk of adverse pregnancy outcome and potentially greater importance 
of angiogenic factors in the pathogenesis of disease in these patients. $^{28}$

The utility of PIGF and other angiogenic factors VEGF, and sFLT-1 in prediction pre-eclampsia is likely limited by the heterogeneity of pathology that underlies the spectrum of clinical presentation of pre-eclampsia. Affected women range from those with early-onset disease and severe intrauterine growth restriction to others with mild symptoms presenting at term. Early, severe disease appears to be more strongly associated with abnormal placentation and abnormalities in angiogenic factors are more pronounced in these patients. Persistently low levels of PIGF throughout pregnancy and abnormal sFLT-1: PIGF ratio identifies a subset of women with an early and more severe presentation of the disease. The use of angiogenic factors may be in categorising pre-eclamptic patients to allow more directed research specific to subtypes of pre-eclampsia.

In women suspected of having pre-eclampsia, but not yet meeting diagnostic criteria, the sFLT-1: PIGF ratio or plasma PIGF alone is useful as a 'rule out' test with a high negative predictive value. Maternal plasma PIGF less than the 5 th centile for gestation at the time of presentation performed better than a 5 factor combination of commonly used clinical parameters (systolic and diastolic blood pressure, alanine transferase, uric acid and dipstick proteinuria) (ROC area 0.87 vs $0.70 P<0.001$ ) in diagnosing women with pre-eclampsia requiring delivery within 2 weeks. ${ }^{29}$ The sensitivity of low PIGF was highest for the delivery of a small for gestational age infant, further supporting the observation that low PIGF reflects placental disease.

Cost-benefit analyses of these tests suggest that angiogenic factor testing will be useful in determining appropriate resource allocation by allowing reduced frequency of observation of women deemed at low risk of developing pre-eclampsia. The sFLT-1: PIGF ratio is also useful for distinguishing patient with preeclampsia from those with conditions that may present similarly such as glomerulonephritis.

\section{TREATMENT OF EXPERIMENTAL MODELS OF PRE-ECLAMPSIA WITH PLGF}

The clinical presentation of pre-eclampsia is a consequence of widespread endothelial dysfunction triggered in part by excess sFLT-1. Binding of local VEGF by sFLT-1 in tissues with high expression of VEGF such as the kidney and liver appears to be responsible for the clinical signs such as proteinuria and raised transaminases. While decreased circulating PIGF does not appear to directly contribute to the clinical syndrome, supplementation of PIGF to correct the angiogenic balance and act as a ligand for excess sFLT-1 has been considered as a possible treatment avenue. Similarly, restoration of the angiogenic factor imbalance by removal of sFLT-1 by apheresis has been attempted with promising results. $^{30}$

There are currently four reports of PIGF treatment in experimental models of pre-eclampsia. Two different rodent models have been successfully treated with exogenous PIGF. Suzuki et al. ${ }^{31}$ established experimental pre-eclampsia by transfection of mice with an adenovirus to increase sFLT-1. Mouse PIGF-2 was given intraperitoneally for two days, which resulted in the reduction of hypertension but not proteinuria. Spradley et al. ${ }^{32}$ and $\mathrm{Zhu}$ et $a \beta^{33}$ used a rat reduced uterine placental perfusion (RUPP) model, which is shown to result in an increase sFLT-1. Spradley et al. administered recombinant human PIGF (rhPIGF) by continuous infusion via an intraperitoneal osmotic pump. This reduced blood pressure, proteinuria and improved glomerular filtration rate in addition to reducing markers of oxidative stress. sFLT-1 levels also decreased significantly. Zhu et al. infused rhPIGF both intravenously and via subcutaneously resulting in blood pressure reduction. Similarly, in a non-human primate uteroplacental ischemic model of pre-eclampsia, the administration of
rhPLGF reduced blood pressure and proteinuria. ${ }^{34}$ The concentration of PIGF decreases in non-human primates with uteroplacental ischaemia but sFLT-1 levels remained elevated in animals that were administered PIGF despite improvement of clinical signs. The conflicting changes in SFLT-1 levels in the RUPP model in rodents as compared with the UPI model in non-human primates may result from differences in ELISA specificity. Commercial kits may measure free sFLT-1 or sFLT-1 bound to VEGF or PIGF. The mouse sFLT-1 kit used by Spradley et al. measures free sFLT-1, suggesting that rhPIGF binds with sFLT-1 and reduces free circulating SFLT-1. ${ }^{32}$ A direct effect of PIGF upon placental or extra-placental release of SFLT-1 remains a possibility.

Pravastatin has been demonstrated to increase PIGF in a lentivirus human sFLT-1 transfected mouse model. ${ }^{35}$ There was reduction in blood pressure and placental and foetal weights normalised. ${ }^{35}$ This may be because of the increase in PIGF; however, pravastatin also has been shown to dampen inflammatory cytokines TNF- $a$ and IL-1 and reduce vascular reactivity which may also account for this observation. At least two clinical trials are currently underway to determine the utility of pravastatin in pre-eclampsia prevention: 'Statins to ameliorate early-onset preeclampsia' (www.controlled-trials.com ISRCTN23410175) and 'Pravastatin for prevention of preeclampsia' (www.ClinicalTrials. gov NCT01717586).

\section{FUTURE DIRECTIONS}

Treatment of pre-eclampsia with PIGF is promising but many uncertainties remain. The delicate balance of anti- and proangiogenic factors, presumably pathological in the case of preeclampsia, may still be significantly disrupted by exogenous PIGF. Although low PIGF concentrations have been identified in women who subsequently develop pre-eclampsia, the normal range of PIGF is wide in healthy pregnant women making interpretation individual cases difficult. The concentration to which PIGF should be restored for individual patients is also uncertain. Timing and duration of exposure to exogenous PIGF for treatment is also important given the usual decline in PIGF concentrations during normal pregnancy.

Off target effects of PIGF are of concern as the VEGFR-1 receptor is present in many tissues. However, as PIGF does not bind to VEGFR-2, it is likely that any unintended effects will not be as significant as compared with VEGF, which causes hypotension and increased vascular permeability mediated by VEGFR-2. Although high circulating PIGF present during pregnancy (as compared to in the non-pregnant state) does not appear to cause abnormalities in other tissues, peripheral administration of PIGF may have untoward effects such as promotion of inflammation and malignancy. A concern would be the effect of this molecule upon the foetus as transgenic mice with PIGF overexpression in T cells displayed impaired angiogenesis, resulting in death during gestation and growth restriction even after birth. ${ }^{11}$

Despite these limitations, restoration of angiogenic factor balance could be a means of treating pre-eclampsia. It has the potential to prolong pregnancy in a condition for which there is currently no definitive treatment excepting delivery of the placenta, even if this means prematurity. Reversal of endothelial dysfunction induced by excess sFLT-1 may be partially achieved by closely titrated replacement of PIGF or removal of SFLT-1 by apheresis. This is a biologically plausible avenue for therapeutic development and needs to be further explored.

\section{CONFLICT OF INTEREST}

The authors declare no conflict of interest. 


\section{ACKNOWLEDGEMENTS}

KC is supported by a National Health Medical Research Council Postgraduate Scholarship GNT1075337.

\section{REFERENCES}

1 Arad A, Nammouz S, Nov Y, Ohel G, Bejar J, Vadasz Z. The Expression of neuropilin-1 in human placentas from normal and preeclamptic pregnancies. Int J Gynecol Pathol 2016.

2 Gobble RM, Groesch KA, Chang M, Torry RJ, Torry DS. Differential regulation of human PIGF gene expression in trophoblast and nontrophoblast cells by oxygen tension. Placenta 2009; 30(10): 869-875.

3 Khaliq A, Dunk C, Jiang J, Shams M, Li XF, Acevedo C et al. Hypoxia downregulates placenta growth factor, whereas fetal growth restriction up-regulates placenta growth factor expression: molecular evidence for 'placental hyperoxia' in intrauterine growth restriction. Lab Invest 1999; 79(2): 151-170.

4 Xu L, Cochran DM, Tong RT, Winkler F, Kashiwagi S, Jain RK et al. Placenta growth factor overexpression inhibits tumor growth, angiogenesis, and metastasis by depleting vascular endothelial growth factor homodimers in orthotopic mouse models. Cancer Res 2006; 66(8): 3971-3977.

5 Van de Veire S, Stalmans I, Heindryckx F, Oura H, Tijeras-Raballand A, Schmidt T et al. Further pharmacological and genetic evidence for the efficacy of PIGF inhibition in cancer and eye disease. Cell 2010; 141(1): 178-190.

6 Lassen U, Chinot OL, McBain C, Mau-Sorensen M, Larsen VA, Barrie M et al. Phase 1 dose-escalation study of the antiplacental growth factor monoclonal antibody RO5323441 combined with bevacizumab in patients with recurrent glioblastoma. Neuro Oncol 2015; 17(7): 1007-1015.

7 Binder NK, Evans J, Salamonsen LA, Gardner DK, Kaitu'U-Lino TJ, Hannan NJ. Placental growth factor is secreted by the human endometrium and has potential important functions during embryo development and implantation. PLOS ONE 2016; 11(10): e0163096.

8 Luna RL, Kay VR, Ratsep MT, Khalaj K, Bidarimath M, Peterson N et al. Placental growth factor deficiency is associated with impaired cerebral vascular development in mice. Mol Hum Reprod 2016; 22(2): 130-142.

9 Dang F, Croy BA, Stroman PW, Figueiro-Filho EA. Impacts of Preeclampsia on the Brain of the Offspring. Rev Bras Ginecol Obstet 2016; 38(8): 416-422.

10 Ratsep MT, Carmeliet P, Adams MA, Croy BA. Impact of placental growth factor deficiency on early mouse implant site angiogenesis. Placenta 2014; 35(9): 772-775.

11 Kang MC, Park SJ, Kim HJ, Lee J, Yu DH, Bae KB et al. Gestational loss and growth restriction by angiogenic defects in placental growth factor transgenic mice. Arterioscler Thromb Vasc Biol 2014; 34(10): 2276-2282.

12 Burke SD, Barrette VF, Bianco J, Thorne JG, Yamada AT, Pang SC et al. Spiral arterial remodeling is not essential for normal blood pressure regulation in pregnant mice. Hypertension 2010; 55(3): 729-737.

13 Athanassiades A, Lala PK. Role of placenta growth factor (PIGF) in human extravillous trophoblast proliferation, migration and invasiveness. Placenta 1998; 19(7): 465-473.

14 Knuth A, Liu L, Nielsen H, Merril D, Torry DS, Arroyo JA. Placenta growth factor induces invasion and activates p70 during rapamycin treatment in trophoblast cells. Am J Reprod Immunol 2014; 73: 330-340.

15 Tayade C, Hilchie D, He H, Fang Y, Moons L, Carmeliet P et al. Genetic deletion of placenta growth factor in mice alters uterine NK cells. J Immunol 2007; 178(7): 4267-4275.

16 Arroyo J, Price M, Straszewski-Chavez S, Torry RJ, Mor G, Torry DS. XIAP protein is induced by placenta growth factor (PLGF) and decreased during preeclampsia in trophoblast cells. Syst Biol Reprod Med 2014; 60(5): 263-273.

17 Desai J, Holt-Shore V, Torry RJ, Caudle MR, Torry DS. Signal transduction and biological function of placenta growth factor in primary human trophoblast. Biol Reprod 1999; 60(4): 887-892.

18 Saffer C, Olson G, Boggess KA, Beyerlein R, Eubank C, Sibai BM. Determination of placental growth factor (PIGF) levels in healthy pregnant women without signs or symptoms of preeclampsia. Pregnancy Hypertens 2013; 3(2): 124-132.

19 Levine RJ, Maynard SE, Qian C, Lim KH, England LJ, Yu KF et al. Circulating angiogenic factors and the risk of preeclampsia. N Engl J Med 2004; 350(7): 672-683.

20 Maynard SE, Min JY, Merchan J, Lim KH, Li J, Mondal S et al. Excess placental soluble fms-like tyrosine kinase 1 (sFlt1) may contribute to endothelial dysfunction, hypertension, and proteinuria in preeclampsia. J Clin Invest 2003; 111(5): 649-658.

21 Poon LC, Zaragoza E, Akolekar R, Anagnostopoulos E, Nicolaides KH. Maternal serum placental growth factor (PIGF) in small for gestational age pregnancy at $11(+0)$ to $13(+6)$ weeks of gestation. Prenat Diagn 2008; 28(12): 1110-1115.

22 Hoeller A, Ehrlich L, Golic M, Herse F, Perschel FH, Siwetz M et al. Placental expression of sFlt-1 and PIGF in early preeclampsia vs. early IUGR vs. age-matched healthy pregnancies. Hypertens Pregnancy 2017; 36(2): 151-160.

23 Mizuuchi M, Cindrova-Davies T, Olovsson M, Charnock-Jones DS, Burton GJ, Yung HW. Placental endoplasmic reticulum stress negatively regulates transcription of placental growth factor via ATF4 and ATF6beta: implications for the pathophysiology of human pregnancy complications. J Pathol 2016; 238(4): 550-561.

24 Tudisco L, Della Ragione F, Tarallo V, Apicella I, D'Esposito M, Matarazzo MR et al. Epigenetic control of hypoxia inducible factor-1alpha-dependent expression of placental growth factor in hypoxic conditions. Epigenetics 2014; 9(4): 600-610.

25 Kleinrouweler CE, Wiegerinck MM, Ris-Stalpers C, Bossuyt PM, van der Post JA, von Dadelszen $P$ et al. Accuracy of circulating placental growth factor, vascular endothelial growth factor, soluble fms-like tyrosine kinase 1 and soluble endoglin in the prediction of pre-eclampsia: a systematic review and meta-analysis. Bjog 2012; 119(7): 778-787.

26 Poon LC, Syngelaki A, Akolekar R, Lai J, Nicolaides KH. Combined screening for preeclampsia and small for gestational age at 11-13 weeks. Fetal Diagn Ther 2013; 33(1): 16-27.

27 Park FJ, Leung CH, Poon LC, Williams PF, Rothwell SJ, Hyett JA. Clinical evaluation of a first trimester algorithm predicting the risk of hypertensive disease of pregnancy. Aust N Z J Obstet Gynaecol 2013; 53(6): 532-539.

28 Kim MY, Buyon JP, Guerra MM, Rana S, Zhang D, Laskin CA et al. Angiogenic factor imbalance early in pregnancy predicts adverse outcomes in patients with lupus and antiphospholipid antibodies: results of the PROMISSE study. Am J Obstet Gynecol 2016; 214(1): 108 e1-108 e14.

29 Chappell LC, Duckworth S, Seed PT, Griffin M, Myers J, Mackillop L et al. Diagnostic accuracy of placental growth factor in women with suspected preeclampsia: a prospective multicenter study. Circulation 2013; 128(19): 2121-2131.

30 Thadhani R, Hagmann H, Schaarschmidt W, Roth B, Cingoez T, Karumanchi SA et al. Removal of soluble fms-like tyrosine kinase- 1 by dextran sulfate apheresis in preeclampsia. J Am Soc Nephrol 2016; 27(3): 903-913.

31 Suzuki H, Ohkuchi A, Matsubara S, Takei Y, Murakami M, Shibuya M et al. Effect of recombinant placental growth factor 2 on hypertension induced by full-length mouse soluble fms-like tyrosine kinase 1 adenoviral vector in pregnant mice. Hypertension 2009; 54(5): 1129-1135.

32 Spradley FT, Tan AY, Joo WS, Daniels G, Kussie P, Karumanchi SA et al. Placental growth factor administration abolishes placental ischemia-induced hypertension. Hypertension 2016; 67(4): 740-747.

33 Zhu M, Ren Z, Possomato-Vieira JS, Khalil RA. Restoring placental growth factorsoluble fms-like tyrosine kinase-1 balance reverses vascular hyper-reactivity and hypertension in pregnancy. Am J Physiol Regul Integr Comp Physiol 2016; 311(3): R505-R521.

34 Makris A, Yeung KR, Lim SM, Sunderland N, Heffernan S, Thompson JF et al. Placental growth factor reduces blood pressure in a uteroplacental ischemia model of preeclampsia in nonhuman primates. Hypertension 2016; 73(4): 330-340.

35 Kumasawa K, Ikawa M, Kidoya H, Hasuwa H, Saito-Fujita T, Morioka Y et al. Pravastatin induces placental growth factor (PGF) and ameliorates preeclampsia in a mouse model. Proc Natl Acad Sci USA 2011; 108(4): 1451-1455.

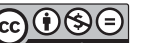

This work is licensed under a Creative Commons AttributionNonCommercial-NoDerivs 4.0 International License. The images or other third party material in this article are included in the article's Creative Commons license, unless indicated otherwise in the credit line; if the material is not included under the Creative Commons license, users will need to obtain permission from the license holder to reproduce the material. To view a copy of this license, visit http:// creativecommons.org/licenses/by-nc-nd/4.0/

(c) The Author(s) 2017 